\title{
El aula invertida como metodología aplicada a estudiantes universitarios en el contexto covid-19
}

\section{The flipped classroom as a methodology applied to university students in the covid-19 context}

Manuel Felipe Guevara Duarez $\mathbb{D D}^{1^{*}}$., Sailor Condezo Tascca ${ }^{2}$. ., Percy Vasquez Duarez ${ }^{5}$ (D) Jhenmy Villarruel Diaz ${ }^{6}$ (D)

\section{RESUMEN}

El presente estudio tiene como objetivo establecer la incorporación del aula invertida en el proceso de enseñanza-aprendizaje de estudiantes universitarios en el contexto de virtualidad ocasionada por el covid-19. Pertenece al enfoque cuantitativo con alcance descriptivo, se trabajó con una muestra de 62 estudiantes universitarios de las Escuelas Profesionales de Derecho y Educación de la Universidad Nacional Amazónica de Madre de Dios que cursan la asignatura de Filosofía en el ciclo académico 2020II, utilizándose como instrumento un cuestionario para el recojo de datos. Los resultados evidencian que la estrategia metodológica del aula invertida repercute en el aprendizaje de manera metacognitiva, siendo también una estrategia innovadora que modifica la forma de aprender tradicional por una forma invertida epistemológica de aprender de los estudiantes; además, el enfoque pedagógico de la enseñanza se traslada al espacio de aprendizaje autónomo-crítico, colaborativo y por competencias, tornándole más dinámico e interactivo donde el docente es el mediador de aprendizajes. Conclusiones, aporta mayor énfasis a la práctica, porque los estudiantes se convierten en protagonistas de su propio aprendizaje sin estar a expensas de los maestros hasta convertirlos en conocimientos.

Palabras clave: Aula invertida, trabajo colaborativo, aprendizaje autónomo, competencias virtuales.

\begin{abstract}
The present study aims to establish the incorporation of the flipped classroom in the teaching-learning process of university students in the context of virtuality caused by covid-19. It belongs to the quantitative approach with descriptive scope, we worked with a sample of 62 university students from the Professional Schools of Law and Education of the National Amazonian University of Madre de Dios who are studying the Philosophy subject in the academic cycle 2020-II, being used as instrument a questionnaire for data collection. The results show that the methodological strategy of the flipped classroom affects learning in a metacognitive way, being also an innovative strategy that modifies the traditional way of learning by an epistemological inverted way of learning of the students; Furthermore, the pedagogical approach to teaching is transferred to the autonomous-critical, collaborative and competency learning space, making it more dynamic and interactive where the teacher is the mediator of learning. Conclusions, provides greater emphasis to practice, because students become protagonists of their own learning without being at the expense of teachers until they become knowledge.
\end{abstract}

Keywords: Flipped classroom, collaborative work, autonomous learning, virtual skills.

DOI: https://doi.org/10.37787/pakamuros-unj.v8i4.145

Recibido: 01/11/2020. Aceptado: 15/12/2020

* Autor para correspondencia

1. Universidad Nacional Mayor de San Marcos, Lima- Perú, mguevarad@unmsm.edu.pe; sailor.condezo@unmsm.edu.pe ; percy.panez@unmsm.edu.pe; jorge.saldana4@unmsm.edu.pe ; vasquez@unmsm.edu.pe

2. Universidad Nacional Amazónica de Madre de Dios, Perú, jvillarruel@unamad.edu.pe 


\section{INTRODUCCIÓN}

En la actualidad la educación universitaria tiene el soporte digital y pedagógico (Salas y Lugo, 2019; Garrote y otros, 2018;), generado por una coyuntura de pandemia mundial (Jin et al., 2020; Li et al., 2020), en el que los cambios educativos demandan mayor protagonismo de los estudiantes en el desarrollo de la enseñanza-aprendizaje, donde se consideren como propósito estimular creatividad, curiosidad y hasta mayor trabajo colaborativo-cooperativo, como también adquirir conocimientos más allá del aula (Flores y otros, 2016).

El trabajo pedagógico de los profesores durante y después de clases de seguro permiten fortalecer y llevar conocimientos a los estudiantes; generar el establecimiento de mejoras y la creación de escenarios educativos con calidad (Godoy et al. 2016). Tiempos en que las herramientas digitales están propiciando cambios radicales durante el desarrollo de actividades académicas. Se observa que, la educación está de manera insoslayable inmersa en el empoderamiento digital de las plataformas virtuales (Hernández \& Tecpan, 2017).

En este devenir, el alcance de la tecnología sumado a los cambios que necesita tomar la educación, las universidades han diseñado e implementando nuevas metodologías pedagógico-didácticas como el aula invertida, con el propósito de desarrollar aprendizajes más allá clases convencionales, sino generar mayor responsabilidad y preparación de parte de los estudiantes en la adquisición de sus propios aprendizajes. En esta perspectiva, germina como alternativa el modelo denominado aula Invertida, originado como Flipped Classroom (Lage, Platt \& Treglia, 2000). Planteamiento original de Jonathan Bergmann y Aaron Sams, profesores en Woodland Park High School, Colorado-EEUU. Quienes iniciaron esta estrategia educativa para que los estudiantes no perdieran clases, lleven material didáctico a casa: audios, videos, lecturas, se preparen y de vuelta al aula, física/virtual, cuenten con una base epistémica que quiera convertirse en conocimiento

Esta propuesta metodológica pretende invertir las actividades realizadas habitualmente en el aula por otras que favorezcan el aprendizaje autónomo y fortalecer los entornos colaborativos. Desde esta óptica, este artículo tiene el propósito de establecer el impacto metacognitivo de utilizar la metodología aula invertida para adquirir aprendizajes significativos, destacar el aprendizaje autónomo, generar aprendizaje colaborativo y aprovechar el aprendizaje por competencias, en pleno contexto virtual de la enseñanza universitaria.

\section{MATERIALES Y MÉTODOS}

Los procesos metodológicos de la investigación están dentro de un enfoque cuantitativo, de alcance descriptivo "porque pretende medir o recoger información de manera independiente o conjunta sobre los conceptos o variable(s) a los que se refiere" (Hernández \& otros, 2014, p. 93); además describe, analiza 
e interpreta sistemáticamente un conjunto de hechos. Estudia el fenómeno en su estado actual y en su forma natural (Sánchez \& Reyes, 2002). Pertenece a un diseño en el que la relevancia metodológica guía y orienta la conducción del proceso de investigación, teniendo en cuenta "la formulación del problema, la hipótesis y el logro de los objetivos de investigación, en el contexto social o natural donde se presenta o identifica la situación problemática" (Carrasco, 2006, p. 58-59). La muestra lo conforman 62 estudiantes universitarios de las Escuelas Profesionales de Derecho y Educación de la Universidad Nacional Amazónica de Madre de Dios en la asignatura de Filosofía del ciclo académico 2020-II.

Para recoger la percepción de las experiencias desarrolladas mediante las clases aplicando la estrategia aula invertida, se procedió la técnica de cuestionario de encuesta, detectándose hallazgos de suma relevancia que grafican epistemológicamente que esta forma de enseñanza permite mejorar los aprendizajes en la Asignatura de Filosofía. Tal como se puede analizar en el cluster (Figura 1 y 2).

\section{RESULTADOS}

Se demuestran resultados del estudio respecto de la participación de 62 estudiantes matriculados en la asignatura de Filosofía de dos escuelas profesionales: Derecho y Educación. Participaron 36 mujeres varones $(58,2 \%)$ y 26 varones (42,8\%); todos ellos adeptos de la tecnología (Guevara et al., 2020) que demostraron conocer recursos tecnológicos y estaban empezando a familiarizarse con recursos virtuales para el desarrollo de clases; indagación importante que buscaba esta investigación.

Luego de aplicar el instrumento de investigación a la muestra de estudio sobre su participación en la asignatura por medio de la estrategia aula invertida. Se empleó un instrumento dividido en tres dimensiones, postulado mediante 15 preguntas, de un instrumento validado y confiable (Alfa de Cronbach 0, 888 con 15 muestras) que recogen aspectos referidos a la estrategia metodológica aula invertida, el nivel de acuerdo o desacuerdo con la propuesta pedagógicas, la utilidad de dicha estrategia a partir de la enseñanza virtual.

Se partió del análisis de observar los análisis de la variable Aula Invertida, Figura 1, donde según los resultados se puede observar que, del 58,2\% son estudiantes mujeres, las mismas que manifiestan que están a gusto con la propuesta metodológica y el $42.8 \%$ son estudiantes varones. Lo que resulta del análisis estadístico del clúster, que las mujeres son las que demuestran mayor responsabilidad y que más trabajan el aula invertida para mejora de sus aprendizajes. 


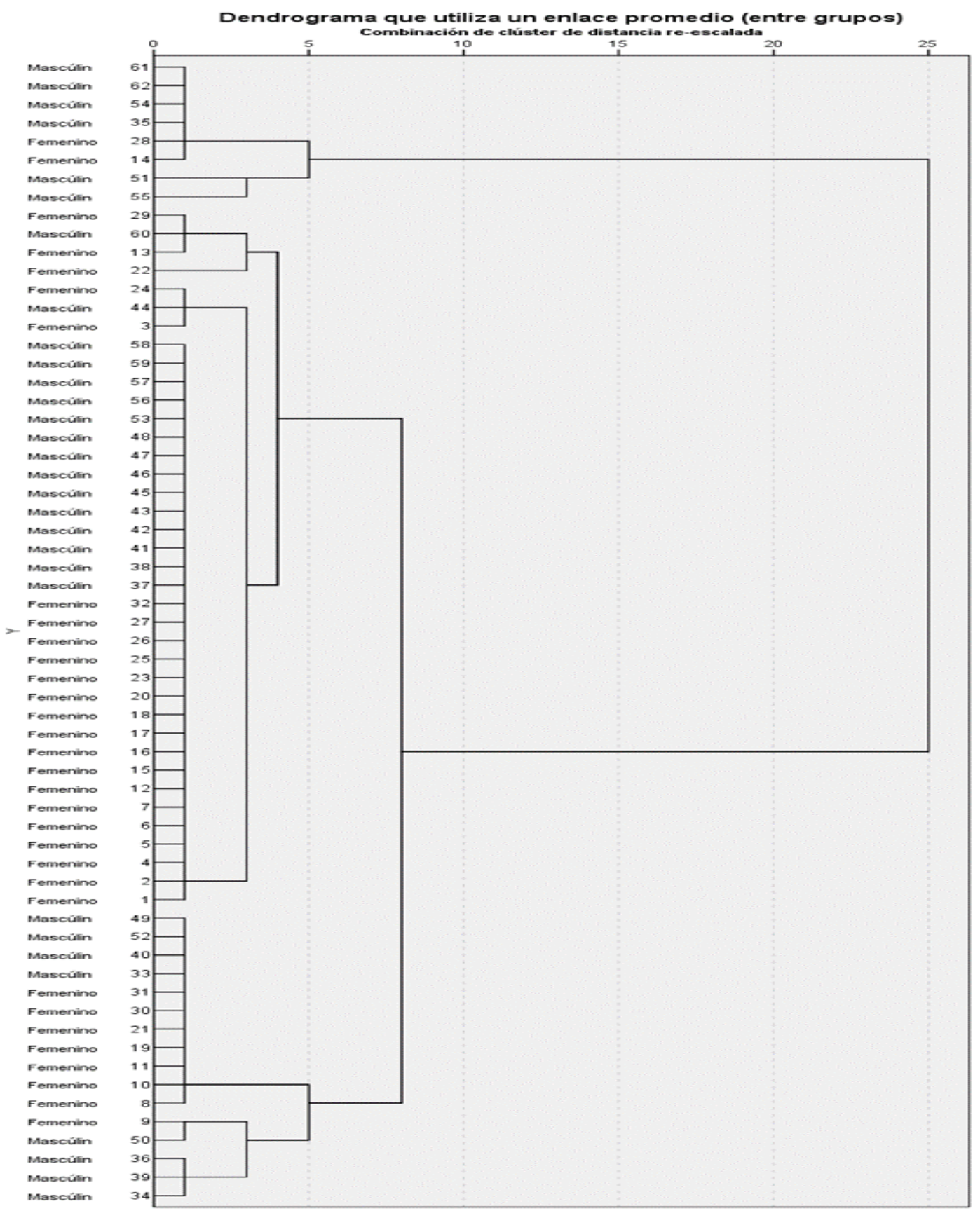

Figura 1. Dendrograma de la variable Aula Invertida. De acuerdo al Sexo.

Universitarios por aprender mediante el aula invertida.

Cabe resaltar que resulta destacado la integración de las tecnologías en los procesos formativos no sólo porque es una forma descentralizadora de generar aprendizajes (Guevara et al., 2020) e implican que los docentes se actualicen e implementes herramientas acordes a la virtualidad (Vertiz et al. 2020 ), superar muros que afectan el ecosistema funcional de la educación superior con el uso adecuado de las tecnologías (Chasín et al. , 2020) en la educación; y sobre todo, se debe promover que los estudiantes 
regulen y manejen los aprendizajes mediante clases y tareas asincrónicas por sus propios medios con fines de descubrimientos autónomos. De no hacer motivador el apoyo de la virtualidad caeríamos en incertidumbre (Monasterio \& Briseño, 2020) del tipo de educación que se debe ejecutar en el contexto de pandemia.

Respecto a las dimensiones, aprendizaje autónomo, como parte de la metodología del aula invertida, en la Figura 2, resulta del análisis estadístico, tener evidencia empírica para señalar destacados niveles de preferencia de parte de los estudiantes universitarios para lograr aprendizaje autónomo mediante el aula invertida.

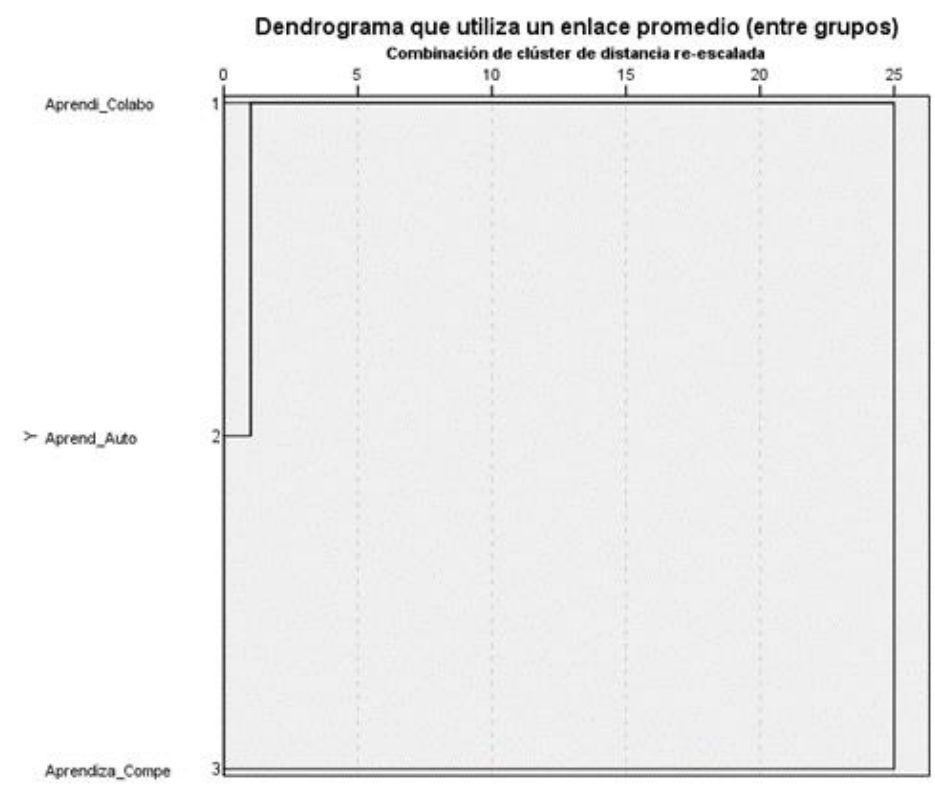

Figura 2: Dendrograma de las dimensiones de la variable Aula Invertida.

En contraste con los resultados obtenidos, tenemos el fundamento de García, \& De la Cruz (2014) que considera los materiales didácticos en educación superior como recurso oportuno para el aprendizaje con autonomía e independencia cognoscitiva del estudiante; apoyado de las Tic en el aula invertida promueve pensamiento crítico, (Cano \& Gonzales, 2016) lo que beneficia en gran medida a reducir los diferentes problemas académicos que antes de la práctica pedagógica de ayudarse con las Tics y aplicar el aula invertida los tenían; las evidencias empíricas de la mejora en los aprendizajes han permitido fortalecer los conocimientos de los estudiantes. Lo que evidencia que los estudiantes dan gran importancia a promover aprendizajes con autonomía en el aula invertida.

Respecto a la dimensión dos, aprendizaje colaborativo como parte de la metodología aula invertida, en la Figura 2, se evidencia, luego del resultado estadístico, señalar destacados niveles de preferencia de parte de los estudiantes universitarios sobre desenvolverse mediante trabajo colaborativo en el aula invertida. Este a forma de adquirir aprendizajes demuestra mayor interés de un gran número de estudiantes universitarios. 
Se destaca que la Metodología Aula Invertida no se aplica sola, sino que cuenta como herramienta estratégica al trabajo colaborativo entre los estudiantes. Éste mediado permite la participación interactiva didáctica en el aula (Guevara, 2014) donde desarrollan habilidades de trabajo en equipo (Gaviria et al, 2019). Así, en el aula se realizan actividades grupales para resolución de problemas y abordaje (Sánchez et al., 2019) de los contenidos temáticos de la asignatura, discutiendo en espacios de socialización hasta llegar a decisiones y conclusión de resultados sobre el tema en común, es en estos momentos cruciales para surgir el fenómeno mental del aprendizaje que interviene el profesor para ordenar informaciones, posibles conocimientos, retroalimentar la forma en que se procedió con la solución de tareas dejadas para trabajar en casa; El docente rescata de entre todas las ideas y discusiones colaborativas surgidas en el clima del aula y define conocimientos. Resultado epistemológico: los alumnos aprendieron. Entonces, se evidencia que sirvió el material, como parte de la enseñanza, que recomendó el profesor para que lo estudiaran en casa, lo lean y analicen, hasta llevarlo a clases y socializarlo.

Esto refiere que las actividades que se realizan en casa son las que se ubican ahora en los niveles más bajo (Hernández \& Tecpan 2017) desde la perspectiva graficada de la taxonomía de Bloom (Figura 3), mientras que las actividades que implican mayor esfuerzo cognitivo se suelen estar en el salón de clases con la mediación del docente (Zainuddin \& Halili, 2016).

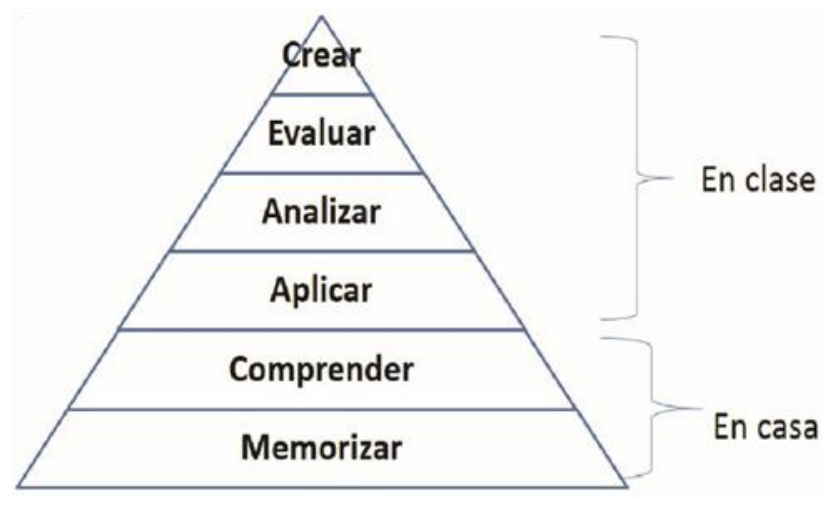

Figura 3. Taxonomía de Bloom en el aula invertida

Se debe diferenciar que en el ambiente tradicional el trabajo del estudiante suele ser individual, competitivo o pasivo, mientras que en el ambiente invertido generado por la virtualidad potencia el trabajo colaborativo propicia un aprendizaje activo. Los estudiantes cuentan con textos o ejercicios propuesto por el docente, que les ayudará a comprender y hasta memorizar información para luego discutirlo en clase.

Además, al analizar la dimensión tercera, aprendizaje por competencias, como parte de la metodología aula invertida, en la Figura 2, se evidencia poco interés el tipo de aprendizaje que se genera en lapráctica 
del aula invertida. Así lo demuestra los resultados estadísticos en la que los estudiantes universitarios suelen tener para desarrollar aprendizajes invertidos.

Se tiene evidencias epistemológicas que en la Metodología Aula Invertida ejecuta acciones pedagógicasdidácticas que promueven aprendizaje por competencias en los estudiantes universitarios. En este sentido, (Gaviria et al, 2019) sustenta que luego de la aplicación del aula invertida en el cuerpo estudiantes se evidencia que la participación en el modelo aula invertida ayuda mejorar la comprensión de los conceptos abordados y desarrollo de competencias que incrementan su propio aprendizaje. Promover el desarrollo de aprendizaje con soporte de las TIC como herramientas para el desarrollo de la competencia intercultural (Garrote, Arenar \& Jiménez, 2018) consolidan el alcance empírico que se recoge en esta propuesta meta educativa.

El giro metodológico-didáctico de lo invertido entonces toma su propia fuerza pedagógica. La instrucción primero, pero en casa y las tareas después, pero en el desarrollo de clase. Los pasos pedagógicos son los mismos; el estudiante ya va a clases con una base epistemológica de la materia, lo que lleva a tener un cambio significativo, dado que las tareas se discuten en un ambiente colaborativo, conducido por el docente, con un papel de mediador activo y participativo en la asignatura (Fidalgo et al., 2017; Fidalgo et al., 2018). En la clase presencial o virtual expresa lo que ha comprendido, lo pone a debate o plantea interrogantes para comprobar si la información que leyó es cierta. Analiza los comentarios e intervenciones de los compañeros y del profesor. Al hacerlo está evaluando que debe quedarle como aprendizaje y termina creando el suyo.

\section{DISCUSIÓN}

El contexto de la educación universitaria está llevándose mediante el soporte tecnológico, el aula física es ahora una plataforma virtual, desarrollándose clases sincrónicas y asincrónicas, y el reto de que la educación universitaria es un problema público y hasta privado, en cuanto la implementación total de las competencias digitales y capaciten el material humano para la educación virtual (Martínez \& Garcés, 2020) durante la pandemia covid-19; a la luz de los resultados obtenidos se evidenció que el aula invertida refleja mejoras sustantivas en el aprendizaje, (Guevara et al., 2020; Salazar, 2019) mediadas por las tecnologías (Tabla 1), haciéndolo de manera positiva (Figura 1), proporcionando más evidencia científica de la que se tiene a la actualidad (Vértiz et al., 2020; Monasterio, \& Briceño, 2020). No obstante, lo destacable de este estudio es que cuando se desagregaron los análisis por dimensiones transformadas en hipótesis descriptivas de la variable aula invertida se evidenció que, para los estudiantes universitarios, hubo una relevancia significativa y metacognitiva del Aprendizaje de manera autónoma (Tabla 2) puesto que beneficia al desarrollo pensamiento crítico, (Cano \& Gonzales, 2016) y reducir los diferentes problemas académicos; 
asimismo, esta metodología didáctica asume el trabajo colaborativo como incidente al combinar la comprensión de lo que leen con las habilidades colaborativa (Gaviria et al, 2019); Además, de tener repercusión estupenda en desarrollar el aprendizaje por competencias (Garrote, Arenar \& Jiménez, 2018) que consolidan la gestión de aprendizaje invertidos (Fidalgo et al. 2017, 20181) lo que lleva a concretar el alcance empírico y epistemológico de los aprendizajes reflejados en conocimientos. Esta propuesta metodológica, por lo tanto, resulta trascendente implementarla en la enseñanza universitaria por la efectividad y significancia que demuestra, puesto que con ello se estaría corroborando, en cierta medida, la efectividad en hacer realidad los aprendizajes, un logro importante dentro de estos escenarios universitarios.

\section{Tabla 2. Del aula Invertida al Aprendizaje Invertido}

\begin{tabular}{ll}
\hline \multicolumn{1}{c}{ Fortalezas/ventajas } & \multicolumn{1}{c}{ Exigencias/Retos } \\
\hline Los conocimientos se desarrollan fuera de tiempo clases & $\begin{array}{l}\text { Aumento de trabajo para los docentes. Preparar tareas que } \\
\text { Estimula el estudio continuo de los estudiantes }\end{array}$ \\
$\begin{array}{l}\text { Asume la información con prospectiva metacognitiva. } \\
\text { Beneficia en el aprendizaje autónomo }\end{array}$ & Demanda el trabajo continuo de los docentes. \\
El aula virtual-física se personifica y enriquece de & Plantearse el reto de alcanzar aprendizajes significativos. \\
interacciones pedagógicas de los mismos estudiantes. & ¿Es sana la interacción colaborativa con los compañeros \\
Asume la información con prospectiva metacognitiva. & para consolidar los aprendizajes? \\
Utiliza el tiempo de clase para realizar evaluación & Planificación de actividades productivas \\
formativa y retroalimentación & Asumir las exigencias como posibilidades de aprendizaje \\
Los trabajos se discuten, corrigen en tiempo de clase y & ¿Cómo se logra que la evaluación formativa sea \\
se transforman en conocimientos. & productiva? \\
& Planificación de métodos de evaluación mediante la \\
& retroalimentación \\
& Se plantean tareas que atraigan el interés de los estudiantes \\
\hline
\end{tabular}

\section{CONCLUSIONES}

La implementación del modelo aula invertida repercute de manera positiva en el aprendizaje de los estudiantes de manera eficaz (Salas \& Lugo, 2019). Esto se puede lograr mediante capacitaciones e investigaciones epistemológicas que permitan guiar a los docentes en la aplicación del aprendizaje invertido. La coyuntura educativa de virtualidad (Vértiz, et al., 2020) es invertida al de la presencialidad, queda hacer seguimiento a los indicadores de mejora a obtenerse mediante la aplicación del aula invertida donde los estudiantes terminen adecuándose, a pesar de las limitaciones tecnológicas y posibilidades sociales a la educación tecnológica. El aula invertida promueve aprendizaje por competencias (Gaviria et al, 2019) que les permite mejoras en la comprensión académica, método apropiado que demanda una planificación apoyada en las tecnologías emergentes. Se inviertes las tareas (actividades) en la secuencia pedagógica-didáctica respecto al sistema tradicional pero el significado es el mismo, fortalecido con el 
trabajo cooperativo y la autonomía para desarrollar pensamiento crítico, los resultados metacognitivos son óptimos en el aprendizaje, que se convierte en una nueva forma de aprender.

\section{AGRADECIMIENTOS}

A los estudiantes de la Universidad Nacional Amazónica de Madre de Dios por aportar al presente estudio.

\section{REFERENCIAS BIBLIOGRÁFICAS}

Barros, V., \& Calero, M. M. (2018). Aula invertida en la enseñanza de Álgebra en la educación superior. Espirales revista multidisciplinaria de investigación, 2(13). https://bit.ly/3nYtIVo

Carrasco Díaz, S. (2006). Metodología de la investigación científica. Lima: San Marcos.

Cano Rodríguez, G. M., \& González Guzmán, J. (2016). ConTIC aprendí: Aula invertida como modelo para promover el pensamiento crítico en estudiantes de grado noveno del Colegio Antonio García. IED (Master's thesis, Universidad de La Sabana). https://bit.ly/37TFv0S

Chacín, A. J. P., González, A. I., \& Peñaloza, D. W. (2020). Educación superior e investigación en Latinoamérica: Transición al uso de tecnologías digitales por Covid-19. Revista de ciencias sociales, 26(3), 98-117.

Fidalgo-Blanco, A., Martinez-Nuñez, Borrás-Gene, \& Sanchez-Medina (2017) Micro Flip Teaching

Fidalgo-Blanco, A., Martinez-Nuñez, M., Borrás-Gene, O., \& Sanchez-Medina, J. J. (2017). Micro flip teaching - An innovative model to promote the active involvement of students. Computers in Human Behavior, 72. https://doi.org/10.1016/j.chb.2016.07.060

Fidalgo-Blanco, Á., Sein-Echaluce, M. L., \& García-Peñalvo, F. J. (2018). Del método de aula invertida al aprendizaje invertido. DOI. 10.5281/zenodo.2081943

García Hernández, I., \& De la Cruz Blanco, G. D. L. M. (2014). Las guías didácticas: recursos necesarios para el aprendizaje autónomo. Edumecentro,6(3), 162-175. ISSN 2077-2874. https://bit.ly/375ahon

Garrote, D., Arenar, J. \& Jiménez, S. (2018). Las TIC como herramientas para el desarrollo de la competencia intercultural. EDMETIC, Revista de Educación Mediática y TIC, 7(2), 166-183. doi: https://doi.org/10.21071/edmetic.10543

Gaviria Rodríguez, D., Arango Arango, J., Valencia Arias, A., \& Bran Piedrahita, L. (2019). Percepción de la estrategia aula invertida en escenarios universitarios. Revista mexicana de investigación educativa, 24(81), 593-614. https://bit.ly/2JRy7en 
Guevara, M. et al. (2020). La educación universitaria en la era del hombre tecnológico: ¿Quo Vadis?. Revista Científica Pakamuros, 8(2), 14 - 24. https://doi.org/10.37787/pakamurosunj.v8i2.124

Guevara Duarez, M. F. (2014). Estrategias de Aprendizaje Cooperativo y Comprensión Lectora con textos filosóficos en Estudiantes de Filosofía de la Facultad de Educación de la Universidad Nacional Amazónica de Madre de Dios, año 2012. (Tesis de postgrado) Universidad Nacional Mayor de San Macos. https://bit.ly/3oH0Kd0

Hernández-Silva, C., \& Tecpan Flores, S. (2017). Aula invertida mediada por el uso de plataformas virtuales: un estudio de caso en la formación de profesores de física. Estudios pedagógicos (Valdivia), 43(3), 193-204. http://dx.doi.org/10.4067/S0718-07052017000300011

Hernández, R; Fernández, C y Baptista, P. (2014). Metodología de la Investigación. México, D.F., Mc Graw Hill.

Jin, Y., Yang, H., Ji, W., Wu, W., Chen, S., Zhang, W., \& Duan, G. (2020). Virology, epidemiology, pathogenesis, and control of covid-19. Viruses, Vol. 12. https://doi.org/10.3390/v12040372

Lage, M. J., Platt, G. J., \& Treglia, M. (2000). Inverting the classroom: A gateway to creating an inclusive learning environment. The Journal of Economic Education, 31(1), 30-43. https://bit.ly/39wFnXQ

Li Y, Liu S, Zhang S, Ju Q, Zhang S, Yang Y, Wang H. (2020) Currenttreatment approaches for COVID19 and the clinical value of transfusion-related technologies, Transfusion and Apheresis Science. https://doi.org/10.1016/j.transci.2020.102839

Lopez, A. (2014). El Aula invertida otra forma de enseñar y aprender. https://bit.ly/35MWO43

Prieto Martín, A. (2017.). Flipped Learning. Aplicar el modelo de aprendizaje inverso. Madrid: Narcea.

Martínez-Garcés, J., \& Garcés-Fuenmayor, J. (2020). Competencias digitales docentes y el reto de la educación virtual derivado de la covid-19. Educación y Humanismo, 22(39), 1-16. https://doi.org/10.17081/eduhum.22.39.4114

Monasterio, D., \& Briceño, M. (2020). Educación mediada por las tecnologías: Un desafío ante la coyuntura del Covid-19. Observador del Conocimiento, 5(1), 100-108.

Mazon, M., de Souza, M. V., \& Spanhol, F. (2016). A sala de aula invertida como modelo para aprendizagem colaborativa: ferramentas e possibilidades na educação superior. Criar Educação.

Salas-Rueda, R.A., \& Lugo-García, J.L. (2019). Impacto del aula invertida durante el proceso educativo superior sobre las derivadas considerando la ciencia de datos y el aprendizaje automático. EDMETIC, Revista de Educación Mediática y TIC, 8(1), 147-170 doi: https://doi.org/10.21071/edmetic.v8i1.9542 
Salazar, C. G., López, Y. P., \& Medina, J. N. (2018). La aplicación del aula invertida como propuesta metodológica en el aprendizaje de matemática. Espíritu Emprendedor TES, 2(1), 1-12. https://doi.org/10.33970/eetes.v2.n1.2018.33

Salazar Jiménez, J. C. (2019). Aula invertida como metodología educativa para el aprendizaje de la química en educación media (Doctoral dissertation, Universidad de la Costa). https://bit.ly/3nJKPtV

Sánchez Rivas, E., Sánchez Rodríguez, J., \& Ruiz-Palmero, J. (2019). Percepción del alumnado universitario respecto al modelo pedagógico de clase invertida. Magis, Revista Internacional de Investigación en Educación, 11(23), 151-168. https://doi.org/10.11144/Javeriana.m1123.paur

Sánchez Carlessi, H. y Reyes, C. (2002). Metodología y diseños de investigación científica. Universidad Ricardo Palma.

Vértiz-Osores, R. I., Jiménez, O. C. S., Herrera, T. A. L., Orué, L. A. M., \& Duarez M. F. G. (2020). El Hombre bajo la apariencia del Homo tecnologicus en el contexto del COVID-19 en Perú. Alpha Centauri, 1(1), 25-37.

Vértiz Osores, J.J., Cucho Flores, R., Vértiz-Osores, R., Vílchez Ochoa, G., \& Angulo Romero, A. (2020). Virtual university education in the context of the health emergency due to COVID19: Challenges in the evaluation processes. International Journal of Early Childhood Special Education (INT-JECSE), 12(1): 467-477. Doi.10.9756/INT-JECSE/V12I1.201027.

Zainuddin, Z., \& Halili, S. H. (2016). Flipped classroom research and trends from different fields of study. International Review of Research in Open and Distributed Learning, 17(3), 313-340. https://bit.ly/2VXQVem 